\title{
Medios digitales y COVID-19: sobreinformación, polarización y desinformación
}

\author{
Digital Media and COVID-19: overinformation, \\ polarization and misinformation
}

\author{
Esteban Zunino \\ CONICET / Universidad Nacional de Cuyo (UNCuyo) \\ estebanzunino@gmail.com \\ https://orcid.org/0000-0002-2951-9872
}

\begin{abstract}
Resumen
El presente trabajo analiza el tratamiento noticioso de la COVID-19 en los principales medios digitales de la Argentina. La pandemia por SARS-CoV-2 reconfiguró, junto con las relaciones económicas y sociales, la centralidad del sistema mediático y la composición temática de sus agendas. La necesidad de información de calidad en tiempo real potenció el rol de los medios digitales como fuentes confiables. En términos específicos se pretende investigar: 1) la composición temática de las agendas informativas durante el período de aislamiento social; 2) el uso de las fuentes de información; y 3) el nivel de crédito diferencial que obtuvieron los actores en el tratamiento informativo. Con ese objetivo, se desarrolló un análisis de las noticias más relevantes de las agendas digitales de Infobae, Página/12, La Nación, y Clarín, entre marzo y octubre de $2020(\mathrm{n}=4.240)$. Los resultados evidencian la existencia de agendas temáticas similares y una presencia homogénea de fuentes oficiales en medios con diferentes líneas editoriales. Además, se advierte una cobertura partisana y polarizada que se pone de manifiesto en el crédito diferencial que adquieren en los medios los puntos de vista desplegados por las fuentes estatales oficialistas y opositoras. El tratamiento informativo de la pandemia, a lo largo de un período extenso, siguió un ciclo de sobreinformación, polarización y desinformación, lesivo para la calidad del debate público.
\end{abstract}

\section{Palabras clave}

Pandemia, periodismo, información, medios electrónicos, política, fuentes de información.

Forma sugerida de citar: Zunino, E. (2021). Medios digitales y COVID-19: sobreinformación, polarización y desinformación. Univeristas, 34, pp. 133-154. 


\begin{abstract}
This investigation analyses the media treatment of COVID-19 in main Argentinian digital media. The SARS-CoV-2 pandemic has reconfigured the economic and social life, jointly with the centrality of the media system and the thematic composition of the media agendas. The demand of information during the outbreak enhanced the role of digital media as reliable sources. In specific terms, this work aims to investigate: 1) the evolution of the media agendas during the social isolation period;2) the information sources deployment; and 3) the differential standing obtained by different actors in the media treatment. To this goal, a quantitative content analysis was developed of the most relevant news items in Infobae, Clarín, La Nación and Página/12 between March 20 and October 18, $2020(n=4,240)$. Results show the existence of similar thematic agendas and a homogeneous presence of state sources in media with different editorial positions. Furthermore, there is partisan and polarized coverage, which is evident in the differential standing attributed to the official and opposition state sources in the analyzed media. The media treatment of the pandemic, over an extensive period, followed a cycle of over-information, polarization and misinformation that was detrimental to public debate quality.
\end{abstract}

Keywords

Pandemic, journalism, information, electronic media, politics, information sources.

\title{
Introducción
}

La pandemia causada por COVID-19 fue declarada el 11 de marzo de 2020. Unos días antes se reportaron los primeros casos en América Latina. En la Argentina, el primer paciente fue notificado por la autoridad sanitaria el 3 de marzo. La decisión de la OMS y los efectos devastadores del nuevo coronavirus sobre los sistemas sanitarios más preparados del mundo obligaron a buena parte de los países latinoamericanos a tomar medidas de confinamiento relativamente tempranas con el afán de aplanar la curva de transmisión y fortalecer a las instituciones de salud.

La llegada del virus a la Argentina constituyó un punto de inflexión en la visibilidad del tema en las agendas mediáticas. Con una preocupación social en aumento, el poder político debió expresarse. El presidente Alberto Fernández, luego de decretar la interrupción de la actividad presencial en las escuelas el 16 de marzo, el 19 de marzo anunció la sanción del decreto 297/20, 
que entraría en vigor un día después, por intermedio del cual se estipulaba el inicio de un Aislamiento Social Preventivo y Obligatorio (ASPO) para disminuir la circulación de personas. Días más tarde, el gobierno comunicó una serie de medidas para apoyar económicamente a los sectores más vulnerables y aportes a las empresas destinados a sostener el empleo privado.

Las pandemias causadas por patógenos desconocidos pueden ser tipificadas como "enfermedades emergentes" (Hallin et al., 2020, p. 2). Una de sus características centrales es que "se convierten en objeto de conocimiento público/mediático antes de consolidarse como objeto de conocimiento médico/científico" (p. 2). En este tipo de situaciones los medios adquieren una centralidad inusitada producto de la mayor necesidad de las personas de recibir información actualizada en tiempo real, fundamental para la toma de decisiones (Farré-Coma, 2015; Muñiz \& Corduneanu, 2020).

En ese sentido, la pandemia por COVID-19 cumple con todos los requisitos necesarios para desatar un proceso de pánico moral (Thompson, 2014): se instaura una amenaza, los medios contribuyen con hacerla reconocible, las elites políticas deben pronunciarse y, finalmente, se producen cambios sociales. La situación se inscribe en una "cultura del riesgo" (Beck, 1998) en la que los medios ocupan un lugar determinante en la construcción simbólica de un estado de miedos en el que la amenaza a la vida orienta una percepción de peligro permanente. Dicha cultura puede ser pensada a partir de la existencia de una serie de amenazas objetivas para la integridad que se asocian a una percepción subjetiva del riesgo que encuentra en los medios un agente dinamizador (Hallin et al., 2020). Ambas dimensiones, la objetiva y la subjetiva, pueden coincidir o no (Kessler \& Focás, 2014).

Resulta necesario advertir entonces que, si bien en situaciones de crisis la información se convierte en un bien sustancial para disminuir la ansiedad y el pánico, dependerá de su calidad que sea efectiva para cumplir con su propósito (Casero-Ripollés, 2020). En un ecosistema mediático convergente en el que la dramatización de la noticia y la inmediatez son los dos criterios de noticiabilidad centrales (Martini, 2017) para acaparar la atención de públicos que llegan a la información de manera aleatoria e incidental (Mitchelstein \& Boczkowski, 2017), se ha desarrollado una espiral de producción de altos volúmenes de información (Salaverría et al., 2020) que no siempre satisface los estándares de calidad requeridos para este tipo de situaciones, desencadenando dinámicas de desinformación (Bennett \& Livingston, 2018; Palpan-Guerra \& Munayco, 2015). 
La cobertura noticiosa de la pandemia constituye un proceso productivo activo por parte de los medios de comunicación. Las agendas mediáticas son consideradas por la agenda setting como un listado organizado de temas (issues) que los media proponen para el debate público con ciertas posibilidades de incidencia (McCombs \& Valenzuela, 2014). Estas son el resultado de mecanismos de selección, omisión, jerarquización (Roberts, 2005) y tematización (Luhmann, 1973) de los problemas.

Diferentes estudios en el marco de esta teoría explicaron que los temas más alejados de la experiencia directa, como la pandemia por COVID-19, estimulan la necesidad de orientación mediática de las audiencias (Jablonski \& Daniele, 1998; Lee, 2004; McCombs, 2006). Tales hallazgos son relevantes para explicar la centralidad que adquirieron los portales online durante el proceso que se analiza (Masip et al., 2020; Muñiz \& Corduneanu, 2020).

En ese sentido, la irrupción de una pandemia producida por un patógeno desconocido puede ser considerada como un evento clave (Brosius \& Eps, 1995) que modifica los criterios de selección de los periodistas. Generalmente asociados con accidentes o catástrofes, los eventos clave instituyen prototipos de cobertura que inciden en la noticiabilidad de los acontecimientos y moldean el tratamiento informativo. Ante su presencia, las rutinas productivas se ven condicionadas por tres factores centrales: 1) las características propias de un problema público altamente noticiable que irrumpe dramáticamente e impacta en los procesos de organización del trabajo periodístico; 2) el interés de las audiencias, que se manifiesta en un incremento de la demanda de información (Casero-Ripollés, 2020; Duc-Huynh, 2020; Muñiz \& Corduneanu, 2020); y 3) la presión de las fuentes como elemento determinante de la fisonomía de las agendas mediáticas (Bennett et al., 2007).

Las fuentes de información son definidas como aquellas personas que entran en contacto con los reporteros y que estos incluyen en sus historias ya que les acercan información sobre los hechos, sus contextos y puntos de vista legitimados por la autoridad que les confiere su posición (Gans, 1979). Entre medios y fuentes se desarrollan relaciones transaccionales y simbióticas de dependencia mutua (Bagdikian, 1985), al punto que Gans (1979) utiliza la metáfora de la danza para dar cuenta del modo en que ambos bailarines procuran conseguir acceso al otro.

Las investigaciones más importantes sobre la relación entre medios y fuentes les otorga a estas últimas una supremacía sobre los medios y un amplio poder para moldear las agendas con una versión interesada de la 
realidad (Schlesinger, 1992). Según la hipótesis del Indexing, acuñada por Bennett (1990), los medios suelen indexar un listado de voces autorizadas, usualmente de actores estatales con fuerte institucionalización y poder de generación de información. Esto lleva a Bennett et al. (2007) a concluir que los medios suelen delegar en los gobiernos el rol de controlarse a sí mismos.

Sin embargo, la visibilidad de las fuentes en las noticias no explica per se su capacidad de incidir sobre los enfoques (Charron, 1995). En efecto, según la premisa del standing, las fuentes citadas por los medios pueden obtener diferentes niveles de crédito o rechazo mediático, lo que repercute en la verosimilitud de sus versiones (Ferree et al., 2002; Koziner, 2018). En ese sentido, trabajos previos evidencian que no existen diferencias significativas entre las fuentes más visibles en los medios argentinos, aunque, a partir de sus testimonios, se construyan coberturas contradictorias y polarizadas relacionadas con sus posiciones editoriales (Koziner, 2018; Zunino, 2019). Tal situación, que repercute negativamente en la diversidad y pluralismo de puntos de vista, constituye un límite a la calidad informativa.

\section{Materiales y método}

El objetivo general de este trabajo es analizar el tratamiento informativo de la COVID-19 en los diarios digitales argentinos más leídos. Los objetivos específicos son: 1) identificar el nivel y las variaciones en la relevancia mediática del problema desde el inicio del distanciamiento social; 2) establecer qué tipo de fuentes fueron consultadas por los medios; y 3) determinar el nivel de crédito que lograron las versiones aportadas por los diferentes actores.

En función de los objetivos, se establecieron las siguientes hipótesis:

- H1: Por tratarse de un evento clave, la COVID-19 adquiere una alta relevancia en los medios digitales que presenta variaciones durante el proceso.

- H2: Las fuentes estatales predominan en las noticias.

- H3: El nivel de crédito obtenido por las fuentes depende de su inscripción política en relación con las posiciones editoriales de los diferentes medios.

El trabajo empírico consistió en un Análisis de Contenido Cuantitativo sobre las noticias de portada, es decir, de la primera a la quinta de las homes 
(primer y segundo scroll), de los diarios online más leídos de la Argentina: Infobae, Clarín, La Nación y Página/12. ${ }^{1}$ La recolección de las noticias se hizo en un corte diario a las 9:00 am, horario de alta afluencia de lectores y en el que se asegura que los temas del día están actualizados (Zunino \& Gri1li Fox, 2020).

La técnica de investigación está orientada a analizar los textos informativos, identificar su estructura, investigar sus cimientos, sus componentes y la forma en que estos interaccionan para su funcionamiento simbólico. El analista utiliza herramientas estadísticas para inferir relaciones entre variables cuyo comportamiento se hipotetiza a partir del marco teórico y estado del arte (Neuendorf, 2002). Mediante deducciones lógicas, el investigador establece inferencias sobre la realidad mediatizada y sobre el contexto en que la información es producida (Krippendorff, 1990).

Para este estudio se constituyó un universo de 4240 piezas periodísticas recolectadas a lo largo de 212 días, entre el 20 de marzo, fecha del inicio del aislamiento y distanciamiento social, y el 18 de octubre de 2020, fecha en la que se cumplieron treinta semanas (siete meses) de observación. Luego, se identificaron las piezas relacionadas con la pandemia $(n=2184)$, unidades de análisis de este trabajo, las cuales fueron codificadas en su totalidad.

\section{Fiabilidad}

Cuatro especialistas intervinieron en el proceso de codificación. Para establecer la fiabilidad del trabajo empírico se extrajo de manera aleatoria una muestra del 10\% de la población, consistente en 220 casos, que fueron recodificados. Seguidamente, se aplicó un ensayo de concordancia estadística entre la codificación original y la muestra de contraste mediante el índice de Kappa de Cohen (k), el cual arrojó un valor medio de acuerdo de k=0,897, el cual expresa un nivel alto de fiabilidad para las variables analizadas.

1 Según la consultora COMSCORE, los diarios online más consumidos en el período enero-junio de 2020, en función del promedio de visitantes únicos son: Infobae, 23178 599; Clarín, 23044 227; La Nación, 18421161 y Página/12, 8943953. 


\section{Análisis y resultados}

En relación con los objetivos planteados, en primer lugar, se analizó la relevancia de la COVID-19 en las agendas mediáticas entre el 20 de marzo y el 18 de octubre de 2020. El gráfico 1 muestra la frecuencia del issue en relación con el hueco informativo (Budd, 1964) de las portadas, sitios más visibles de las homes.

En términos agregados, las noticias sobre la pandemia ocuparon el $51,5 \%$ del espacio disponible. Se evidencia la relación de dos variables que permiten inferir una alta relevancia del asunto en los medios. A una alta frecuencia de cobertura del tema, que permite establecer que cinco de cada diez noticias publicadas durante el período fueron sobre la pandemia, se suma que esas piezas informativas se ubicaron en los sitios de mayor jerarquía, como lo son los primeros scrolls. El hallazgo adquiere mayor relevancia si se tiene en cuenta que diferentes estudios demostraron que las portadas resultan indicativas de la composición del hueco informativo total (McCombs, 2006; Zunino \& Grilli-Fox, 2020).

\section{Gráfico 1 \\ Frecuencia de temas de la agenda informativa en el período de aislamiento social por la COVID-19. Infobae, Clarín, Página/12 y La Nación}

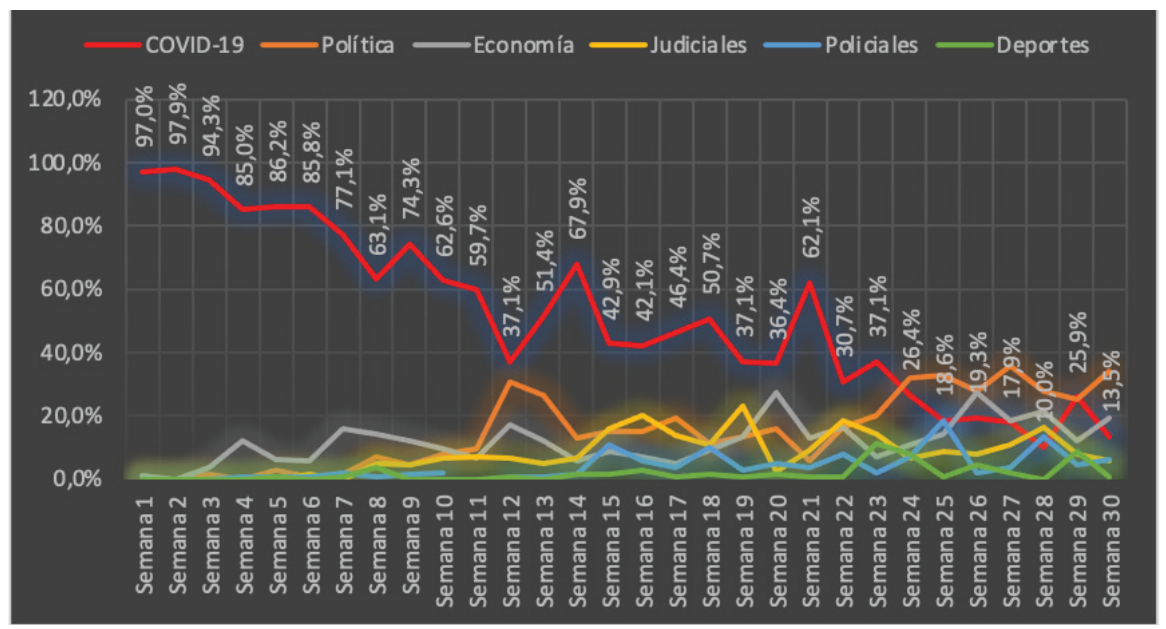

Fuente: Elaboración propia. 
Asimismo, es posible advertir a partir de la Tabla 1, una estructuración temática homogénea entre los diferentes medios analizados. Si bien los máximos niveles de semejanza se registraron entre Clarín y La Nación, existen correlaciones estadísticamente significativas entre los temas dispuestos en las agendas de los cuatro diarios digitales. El hallazgo permite afirmar que los datos del gráfico 1 expresan las variaciones de agendas mediáticas semejantes, aunque se trate de portales con diferentes líneas editoriales.

\section{Tabla 1}

Correlación de Pearson de la composición temática de las agendas mediáticas. Infobae, Clarín, Página/12 y La Nación

\begin{tabular}{|l|l|c|c|c|c|}
\hline \multicolumn{2}{|c|}{} & Clarín & La Nación & Infobae & Página12 \\
\hline \multirow{4}{*}{ Clarín } & Correlación de Pearson & 1 &, $979^{* *}$ &, $861^{* *}$ &, $834^{* *}$ \\
\cline { 2 - 6 } & Sig. (bilateral) & &, 000 &, 000 &, 000 \\
\cline { 2 - 6 } & $\mathrm{N}$ & 1060 & 1060 & 1060 & 1060 \\
\hline \multirow{4}{*}{ La Nación } & Correlación de Pearson &, $979^{* *}$ & 1 &, $898^{* *}$ &, $883^{* *}$ \\
\cline { 2 - 6 } & Significancia &, 000 & &, 000 &, 000 \\
\cline { 2 - 6 } & $\mathrm{N}$ & 1060 & 1060 & 1060 & 1060 \\
\hline \multirow{3}{*}{ Página/12 } & Correlación de Pearson &, $861^{* *}$ &, $898^{* *}$ & 1 &, $839^{* *}$ \\
\cline { 2 - 6 } & Significancia &, 000 &, 000 & &, 000 \\
\cline { 2 - 6 } & $\mathrm{N}$ & 1060 & 1060 & 1060 & 1060 \\
\cline { 2 - 6 } & Correlación de Pearson &, $834^{* *}$ &, $883^{* *}$ &, $839^{* *}$ & 1 \\
\cline { 2 - 6 } & Significancia &, 000 &, 000 &, 000 & 1060 \\
\hline **. Correlación significativa para el nivel 0,01 (BILATERAL). & 1060 & 1060 & 1060 & \\
\hline
\end{tabular}

Fuente: Datos construidos por el autor.

Si se analiza el desarrollo de la agenda mediática a lo largo del período (gráfico 1) resulta posible advertir que la COVID-19 acaparó la atención mediática de modo significativo hasta finales del mes de abril (semana 6), etapa en la que más de ocho de cada diez noticias publicadas fueron sobre el tema. La curva de frecuencia de cobertura a partir de ese momento expresa una tendencia descendente que da cuenta de una caída en la visibilidad del asunto con el correr de las semanas. Se publicaron seis noticias de cada diez 
sobre el tema entre finales del mes de abril y mediados del mes de junio (semana 11), cuatro de cada diez entre los primeros días de junio y la mitad de agosto (semana 21) y no más de dos de cada diez desde la tercera semana del mes de agosto y los últimos días de octubre (semana 30).

El dato llamativo de la evolución temática de las agendas es que se evidencia un pico de sobreinformación acerca de la pandemia en sus inicios, cuando la COVID-19 se constituyó en un monotema mediático, que, sin embargo, llegó al mes de octubre, cumbre de contagios y muertes en el país, con niveles mínimos de cobertura.

\section{Gráfico 2 \\ Evolución de casos de COVID-19. Argentina. \\ 20 de marzo al 18 de octubre de 2020}

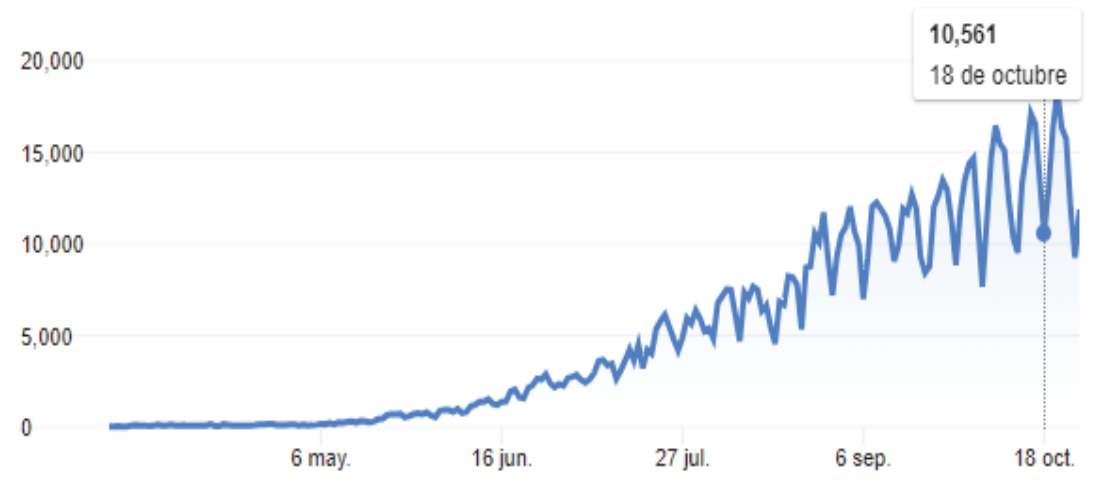

Fuente: Google, a partir de estadísticas de la autoridad nacional de salud.

Varios factores explican la invisibilización de la pandemia en el momento más álgido de la enfermedad. Uno de ellos es la competencia temática con otros asuntos que, al comienzo del período de aislamiento, habían quedado relegados. Por ejemplo, la discusión política, que reapareció en las agendas producto de algunas decisiones tomadas principalmente por el gobierno nacional. El 8 de junio (semana 12) el presidente Alberto Fernández anunció un proyecto de expropiación de la empresa Vicentín, una de las exportadoras de materias primas agropecuarias más importantes del país. La decisión, que fue desestimada días después por el propio gobierno, rompió los precarios 
consensos políticos sobre el manejo de la pandemia que existieron durante las primeras semanas entre oficialismo y oposición. Si bien la polarización venía creciendo por situaciones relacionadas con la gestión del aislamiento social, el anuncio del proyecto de expropiación desató un proceso de enfrentamiento político que llegó a niveles máximos y que reinstaló una agenda de temas independientes de la COVID-19. Ese evento reavivó también la discusión sobre la marcha de la economía en un contexto de profunda crisis. En tanto, la propuesta del Ejecutivo al Parlamento de un proyecto de reforma de la justicia a fines de julio (semana 16), ampliamente criticado por la oposición, instaló la discusión judicial, relacionada con denuncias de corrupción hacia figuras del oficialismo. Finalmente, hacia finales del período observado reaparecieron otros temas habituales de las agendas mediáticas, como lo son los hechos de inseguridad y los deportes, condimentos frecuentes del menú informativo de las épocas pre-pandémicas.

En suma, los datos expresados permiten establecer un ciclo de cobertura informativa que se presenta desacoplado de la evolución de la pandemia. En efecto, mientras que la mayor visibilidad mediática del tema se asoció con la irrupción de la enfermedad en el país, la sobreinformación de los comienzos se condice con un promedio de 150 contagios diarios y menos de una decena de fallecidos por día. En cambio, en la semana de mayor cantidad de infecciones, que se dio al final del período de este trabajo, la frecuencia de cobertura acaparó apenas el 13,5\% del hueco informativo. En ese sentido, es posible afirmar que la evolución del tratamiento mediático resultó inversamente proporcional a la evolución de contagios y muertes.

Los hallazgos permiten analizar que la noticiabilidad de una pandemia no se asocia necesariamente con su gravedad epidemiológica - a nivel nacional e internacional - sino más bien con sus componentes novedosos y noticiables. Prueba de ello es que, en los momentos de mayor incertidumbre sobre el tema, cuando la demanda informativa y desconocimiento se ubicaron en niveles máximos, la cobertura fue total. Con el correr del tiempo, y probablemente producto de la saturación de información y cristalización de cifras de enfermos y muertos, sumado a que la experiencia pandémica cada vez más encontró fuentes intersubjetivas que la alejaron de su condición de tema no experiencial, la cobertura mediática viró de una sobrerrepresentación inicial a una subrepresentación que coincidió con el momento de mayor gravedad objetiva. Ambas situaciones instituyen un ciclo de sobreinforma- 
ción y desinformación favorecido por un clima de fuerte polarización política en el cual se instalaron discusiones que compitieron por la atención mediática en el momento de mayor incidencia de la enfermedad.

Ahora bien, una variable central para analizar los estándares de calidad informativa es la identificación de la presencia y crédito de los diferentes sujetos o instituciones referenciados en las piezas como fuentes informativas.

Un primer hallazgo a tener en cuenta es que la media de fuentes citadas fue de 2.47 por noticia, dato que, si bien no alcanza a las tres fuentes requeridas como requisito básico de triangulación (Rodrigo-Alsina, 1989), se ubica por encima de la media para otro tipo de temas en la prensa digital argentina (Zunino, 2019).

\section{Gráfico 3}

Frecuencia de aparición de fuentes de información durante el ASPO por coronavirus. Infobae, Clarín,

\section{La Nación y Página/12. 20 de marzo al 18 de octubre de 2020}

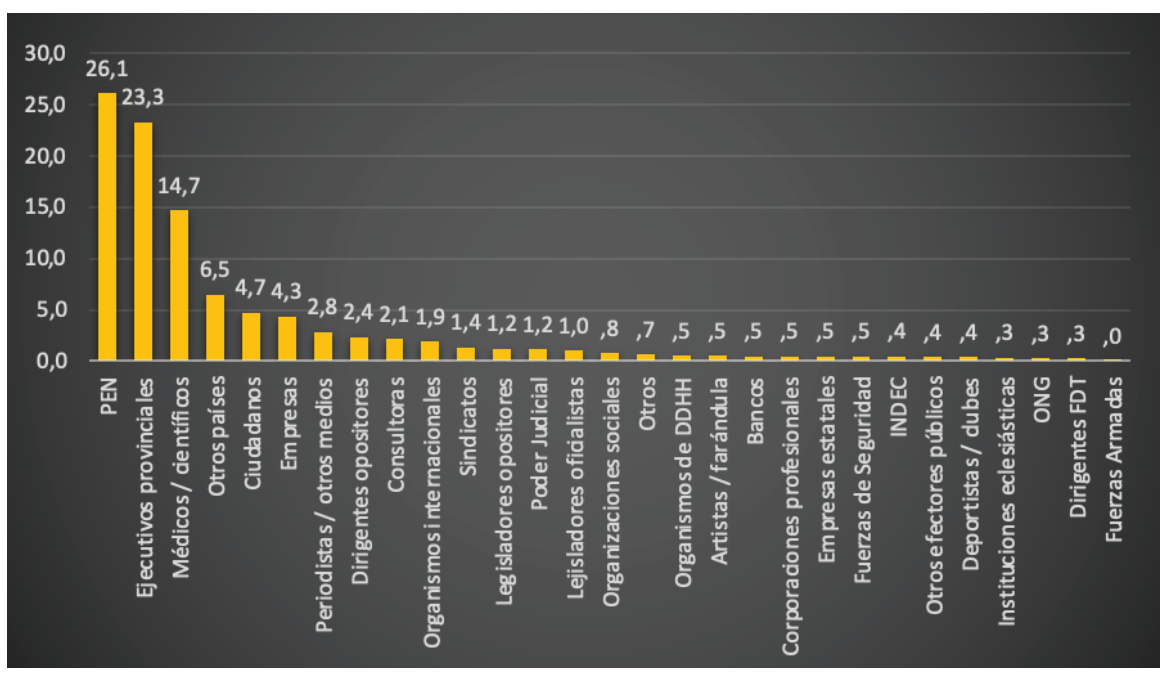

Fuente: Elaboración propia.

El gráfico 3 expresa la presencia de las diferentes fuentes en las agendas informativas. Si se computan de manera agregada, las fuentes oficiales estatales constituyen el $69,1 \%$, mientras que las no oficiales alcanzan el 
30,9\%. El Poder Ejecutivo Nacional fue el actor central en la provisión de datos oficiales sobre la pandemia $(26,1 \%)$, seguido por los gobiernos provinciales $(23,3 \%)$, con especial visibilidad de los de la Ciudad de Buenos Aires $(10,1 \%)$ y de la Provincia de Buenos Aires (7\%). En tanto, la consulta a médicos y científicos especialistas resultó alta respecto de otros asuntos $(14,7 \%)$. Los ciudadanos $(4,7 \%)$ aparecieron generalmente como damnificados por la enfermedad o el aislamiento, mientras que con una menor relevancia incidieron otros actores con alto nivel de institucionalización, como las empresas $(4,3 \%)$, periodistas $(2,8 \%)$, dirigentes opositores $(2,4 \%)$ y consultoras $(2,1 \%)$.

Los hallazgos permiten corroborar para este caso la hipótesis del Indexing, la cual estipula la supremacía de fuentes estatales en las coberturas mediáticas. Ahora bien, el dato reclama ser interpretado en la particularidad de un contexto de pandemia e infodemia.

Los cuatro portales de mayor lectura de la Argentina estructuraron sus coberturas a partir de datos mayormente provistos por agentes estatales que, además de elaborar indicadores oficiales sobre la evolución de la pandemia, fueron quienes decidieron y anunciaron las políticas públicas de restricciones de circulación, ayuda económica y gestión de la crisis. Complementariamente, las voces de médicos o científicos fueron recurrentes en el tratamiento informativo. Por lo tanto, los medios digitales argentinos parecen haber seguido las indicaciones de la OMS respecto del uso de fuentes oficiales y expertas como método para contrarrestar la infoxicación. La afirmación resulta concordante con el rol de curaduría que las audiencias le atribuyeron a los medios tradicionales y/o digitales con trayectorias reconocidas como canales privilegiados de información confiable, en un contexto de saturación informativa y desinformación que expandió la necesidad de chequeo (DucHuynh, 2020; Muñiz \& Corduneanu, 2020; Salaverría et al., 2020).

Adicionalmente, tal como sucedió con la composición temática de las agendas, el comportamiento de los medios analizados respecto del uso de las fuentes de información muestra correlaciones altamente significativas (tabla 2), dato que indica que los periódicos, independientemente de sus líneas editoriales, consultaron a las mismas fuentes y en proporciones similares. 
Tabla 2

Correlación de Pearson fuentes de información citadas. Infobae, Clarín, Página/12 y La Nación

\begin{tabular}{|c|c|c|c|c|c|}
\hline & & $\begin{array}{c}\text { Fuentes } \\
\text { Página/12 }\end{array}$ & $\begin{array}{l}\text { Fuentes } \\
\text { Infobae }\end{array}$ & $\begin{array}{c}\text { Fuentes } \\
\text { La Nación }\end{array}$ & $\begin{array}{c}\text { Fuentes } \\
\text { Clarín }\end{array}$ \\
\hline \multirow{3}{*}{$\begin{array}{l}\text { Fuentes } \\
\text { Página/12 }\end{array}$} & Correlación de Pearson & 1 &, $987^{* *}$ &, $986^{* *}$ &, $968^{* *}$ \\
\hline & Significación & &, 000 & ,000 & 000 \\
\hline & $\mathrm{N}$ & 1271 & 1165 & 1233 & 1271 \\
\hline \multirow{3}{*}{ Fuentes Infobae } & Correlación de Pearson &, $987^{* *}$ & 1 &, $987^{* *}$ & $966^{* *}$ \\
\hline & Significación & 000 & & ,000 & 000 \\
\hline & $\mathrm{N}$ & 1165 & 1165 & 1165 & 1165 \\
\hline \multirow{3}{*}{$\begin{array}{l}\text { Fuentes } \\
\text { La Nación }\end{array}$} & Correlación de Pearson &, $986^{* *}$ &, $987^{* *}$ & 1 &, $987^{* *}$ \\
\hline & Significación & 000 & ,000 & & 000 \\
\hline & $\mathrm{N}$ & 1233 & 1165 & 1233 & 1233 \\
\hline \multirow{3}{*}{ Fuentes Clarín } & Correlación de Pearson &, $968^{* *}$ &, $966^{* *}$ &, $987^{* *}$ & 1 \\
\hline & Significación & 000 & 000 & 000 & \\
\hline & $\mathrm{N}$ & 1271 & 1165 & 1233 & 1381 \\
\hline
\end{tabular}

Fuente: Datos construidos por el autor.

Llegados a este punto, ¿es posible afirmar que la mera consulta a fuentes oficiales en el contexto de una pandemia asegura estándares mínimos de calidad informativa?

La tabla 3 recoge el nivel de crédito (standing) que obtuvieron en cada medio las principales fuentes estatales. Como se dijo, aunque los cuatro portales incluyeron preponderantemente fuentes oficiales, el nivel de crédito y descrédito que les atribuyeron en función de sus orientaciones político/editoriales resulta revelador.

Si bien, en términos generales, los datos oficiales producidos por el Ministerio de Salud no fueron desacreditados, Infobae, La Nación y Clarín expresaron una duda mayor sobre las estadísticas y medidas sanitarias. En cambio, Página/12 nunca puso esos datos en cuestión. Los legisladores de 
la oposición y el Ejecutivo de la Capital Federal, conducido por la coalición política opositora Juntos por el Cambio, resultaron las fuentes más acreditadas por Infobae, Clarín y La Nación, y las que menor nivel de standing obtuvieron en las noticias de Página/12. El presidente Alberto Fernández adquirió niveles de crédito bajos cuando fue citado como fuente en Clarín y $L a$ Nación y un alto volumen de rechazo a sus afirmaciones. En Infobae el nivel de crédito presidencial fue mayor, aunque también lo fue la refutación de sus dichos, lo que evidencia una cobertura más compensada sobre su figura. Contrariamente, la aprobación en Página/12 del primer mandatario fue casi total, con niveles ínfimos de crítica a sus posturas.

\section{Tabla 3}

Standing de las principales fuentes estatales según medio. Infobae, Clarín, Página/12 y La Nación

\begin{tabular}{|c|c|c|c|c|c|c|c|c|c|c|c|c|}
\hline & \multicolumn{3}{|c|}{ Clarín } & \multicolumn{3}{|c|}{ La Nación } & \multicolumn{3}{|c|}{ Infobae } & \multicolumn{3}{|c|}{ Página/12 } \\
\hline & $\begin{array}{c}\text { Se } \\
\text { sostiene }\end{array}$ & $\begin{array}{l}\text { No se } \\
\text { refuta }\end{array}$ & $\begin{array}{c}\text { Se } \\
\text { desa- } \\
\text { credita }\end{array}$ & $\begin{array}{c}\text { Se } \\
\text { sostiene }\end{array}$ & $\begin{array}{l}\text { No se } \\
\text { refuta }\end{array}$ & $\begin{array}{c}\text { Se } \\
\text { desa- } \\
\text { credita }\end{array}$ & $\begin{array}{c}\text { Se } \\
\text { sostiene }\end{array}$ & $\begin{array}{l}\text { No se } \\
\text { refuta }\end{array}$ & $\begin{array}{c}\text { Se } \\
\text { desa- } \\
\text { credita }\end{array}$ & $\begin{array}{c}\text { Se } \\
\text { sostiene }\end{array}$ & $\begin{array}{l}\text { No se } \\
\text { refuta }\end{array}$ & $\begin{array}{c}\text { Se } \\
\text { desa- } \\
\text { credita }\end{array}$ \\
\hline $\begin{array}{l}\text { Legisladores } \\
\text { opositores }\end{array}$ & $88,9 \%$ & $11,1 \%$ & & $57,1 \%$ & $42,9 \%$ & & $80,0 \%$ & $20,0 \%$ & & & $28,6 \%$ & $71,4 \%$ \\
\hline $\begin{array}{l}\text { Ejecutivo } \\
\text { CABA }\end{array}$ & $74,0 \%$ & $19,3 \%$ & $6,6 \%$ & $74,8 \%$ & $23,1 \%$ & $2,0 \%$ & $78,9 \%$ & $13,7 \%$ & $7,4 \%$ & $15,5 \%$ & $64,3 \%$ & $20,2 \%$ \\
\hline $\begin{array}{l}\text { Ministerio de } \\
\text { Salud }\end{array}$ & $73,3 \%$ & $18,7 \%$ & $8,0 \%$ & $73,1 \%$ & $20,2 \%$ & $6,7 \%$ & $84,8 \%$ & $10,1 \%$ & $5,1 \%$ & $64,5 \%$ & $35,5 \%$ & \\
\hline $\begin{array}{l}\text { Ejecutivo } \\
\text { PBA }\end{array}$ & $29,3 \%$ & $40,7 \%$ & $30,1 \%$ & $27,8 \%$ & $38,9 \%$ & $33,3 \%$ & $50,0 \%$ & $26,5 \%$ & $23,5 \%$ & $36,2 \%$ & $58,0 \%$ & $5,8 \%$ \\
\hline $\begin{array}{l}\text { Legisladores } \\
\text { oficialistas }\end{array}$ & $25,0 \%$ & $31,3 \%$ & $43,8 \%$ & $18,8 \%$ & $18,8 \%$ & $62,5 \%$ & $35,0 \%$ & $15,0 \%$ & $50,0 \%$ & $54,5 \%$ & $45,5 \%$ & \\
\hline $\begin{array}{l}\text { Alberto } \\
\text { Fernández }\end{array}$ & $20,6 \%$ & $34,0 \%$ & $45,4 \%$ & $28,4 \%$ & $29,9 \%$ & $41,8 \%$ & $52,6 \%$ & $11,8 \%$ & $35,5 \%$ & $62,3 \%$ & $36,2 \%$ & $1,5 \%$ \\
\hline $\begin{array}{l}\text { Cristina } \\
\text { Fernández de } \\
\text { Kirchner }\end{array}$ & & $20,0 \%$ & $80,0 \%$ & & $33,3 \%$ & $66,7 \%$ & & & $100,0 \%$ & $80,0 \%$ & $20,0 \%$ & \\
\hline
\end{tabular}

Fuente: Elaboración propia

Finalmente, el caso paradigmático de polarización editorial expresado en el crédito o descrédito a las fuentes como mecanismo de posicionamiento mediático se dio respecto de la vicepresidenta Cristina Fernández de Kirchner. Mientras que la expresidenta (2007-2015) acaparó el mayor nivel de standing en Página/12, sin que sus opiniones fueran cuestionadas durante el 
período analizado, la refutación explícita de sus puntos de vista fue total en Infobae y muy alta en Clarín y La Nación, sin que se registren durante todo el recorte temporal casos en los que esos diarios hayan acreditado su palabra.

En soporte de los resultados descriptivos, y con el fin de contrastar las diferencias respecto del nivel de standing entre los medios, se realizó un ANOVA y una prueba HSD Tukey que mostró diferencias estadísticamente significativas entre dos subconjuntos: en el primero se ubicaron Infobae $(1,39$, p. $<0,05)$, Clarín $(1,43$ p. $<0,05)$ y La Nación $(1,47$ p. $<0,05)$, y en el segundo Página/12 (1,71 p. <0,05). Las pruebas, a la vez que confirman las diferencias entre los primeros tres diarios y el último, ratifican la homogeneidad entre los componentes del primer conjunto.

Los resultados expuestos respecto de las fuentes confirman en este objeto de pesquisa la hipótesis del standing. Es decir, a la vez que se constató una presencia homogénea de actores que aportan información a medios con diferentes líneas editoriales, lo que ratifica la noción de que el simple recuento de las fuentes no resulta suficiente para dar cuenta de la influencia que estas adquieren en la construcción de los marcos interpretativos que se despliegan en las noticias, los niveles diferenciales de crédito y descrédito aportan pistas sobre los modos en los que cada medio encuadró el problema. El hallazgo pone en evidencia que, si bien todos los periódicos recurrieron a fuentes oficiales a lo largo de siete meses de pandemia, Clarín, La Nación e Infobae - los tres portales más leídos del país - confrontaron sistemáticamente con los puntos de vista del oficialismo. En cambio, Página/12 expresó una postura contraria a los tres primeros, generalmente acrítica de las versiones oficiales. En suma, el tratamiento mediático de la COVID-19 en los medios más leídos de la Argentina se expresó a partir una composición homogénea de las agendas a nivel temático y de presencia de fuentes de información, aunque fuertemente polarizado en relación con el nivel de crédito y descrédito al que accedieron diferencialmente las versiones oficialistas y opositoras.

\section{Discusión y conclusiones}

El presente trabajo se propuso analizar la cobertura mediática de la Covid-19 en los principales diarios online de la Argentina. Subyacente a ese objetivo se partió de una preocupación central orientada a indagar sobre el papel desempeñado por los medios digitales en un escenario excepcional y 
en el marco de un ecosistema convergente que favorece, vía saturación de contenidos, la desinformación, fragmentación y polarización política (Bennett \& Livingston, 2018).

En primer lugar, se constató que los medios digitales más importantes de la Argentina mantuvieron una agenda temática homogénea que tuvo una particularidad: en el inicio del período de aislamiento social la pandemia acaparó la totalidad de la atención mediática. Tal comportamiento puede explicarse a partir de diferentes variables, entre la que destacan el aumento de la necesidad de orientación y de la demanda informativa de las audiencias (Casero-Ripollés, 2020; Duc-Huynh, 2020; Muñiz, 2020), el pronunciamiento de las elites políticas y el cambio que los eventos clave (Brosius \& Eps, 1995) introducen en las rutinas productivas al modificar los criterios de selección y promover series sobre acontecimientos relacionados.

Sin embargo, luego de una primera instancia de sobrerrepresentación de la enfermedad en un momento en el que el riesgo objetivo de contagio era bajo, con el correr de los meses se generó la contradicción de que en el pico de la pandemia la cobertura mediática fue mínima. En efecto, mientras que la presencia de la COVID-19 acaparaba a más de nueve de cada diez noticias de la agenda durante marzo y abril, cuando los contagios apenas superaban el centenar diario a nivel nacional, hacia el final del período con más de 18000 casos y 400 muertes por día, la presencia del tema en los medios apenas acaparó el 13,5\% del hueco informativo. Las oscilaciones, por lo tanto, estuvieron mucho más asociadas a la novedad de la emergencia, los anuncios gubernamentales relacionados con el uso del espacio público y la gestión económica de la crisis que con la evolución de la enfermedad. En consecuencia, la cobertura mediática mantuvo una relación inversamente proporcional a la dimensión objetiva del riesgo de contagio, pudiendo haber dinamizado procesos de pánico moral (Thompson, 2014) por sobreinformación en momentos de baja transmisibilidad del virus y de desinformación en el momento mayor riesgo objetivo.

Para ahondar sobre este aspecto, el estudio indagó en el uso mediático de las fuentes, factor sustancial de la calidad informativa. Con ese fin se contrastó la hipótesis del Indexing y se comprobó la supremacía de las fuentes estatales en el tratamiento informativo de la pandemia. El hallazgo permite relativizar los planteos de Bennett $(1990,2007)$ sobre lo lesiva que puede resultar su preponderancia en las agendas informativas, ya que en ciertos contextos de crisis y viralización de contenidos apócrifos o inexactos por 
medios conectivos, la consulta de fuentes oficiales en el marco de una enfermedad global resulta saludable. Más aún si se tiene en cuenta que estas estuvieron acompañadas por voces de especialistas, médicos y científicos. Probablemente, producto del trabajo profesional de filtrado o curaduría de la información ejercido por medios con trayectorias consolidadas reconocibles para la población, los diarios digitales se impusieron como fuentes confiables e incrementaron sus niveles de audiencia (Casero-Ripollés, 2020; DucHuynh, 2020; Muñiz, 2020).

No obstante, el estudio de las fuentes se complementó con la indagación sobre otras dimensiones de análisis. En ese sentido, la premisa del standing (Ferree et al., 2002) sostiene que, además de la medición de la presencia de las fuentes en las noticias, resulta necesario investigar qué puntos de vista son acreditados por los medios y cuáles son rebatidos o descartados.

El estudio del crédito diferencial de las fuentes permitió descubrir que en la Argentina existió una cobertura polarizada, partisana y desigual de la pandemia, aun cuando las agendas temáticas y la presencia de las fuentes fue homogénea en medios con diferentes posiciones editoriales. Mientras que Clarín, La Nación e Infobae se mostraron proclives a aceptar o incluso destacar las visiones sobre el problema de los sectores opositores a desacreditar a las principales figuras del PEN, Página/12 tomó las versiones oficiales sin objetarlas. Dicho comportamiento informativo tiende a la construcción de representaciones fragmentadas, descontextualizadas y simplificadoras de una realidad compleja y dramática que, a la vez que restringe la disponibilidad de insumos informativos diversos y plurales para la toma de decisiones, repercute negativamente en la posibilidad de hallar en un mismo medio una gama razonable de puntos de vista que robustezca el debate público.

Así, la mediatización de la pandemia siguió un ciclo que, en términos conceptuales, podría plantearse en tres etapas. A un primer momento signado por una saturación informativa - que podría haber generado, por exceso, pánico (Thompson, 2014), desinformación y cinismo (Cappella \& Jamieson, 1996) - le siguió un segundo momento de recrudecimiento de la polarización política - y mediática - que dio lugar a una última fase de alta competencia temática que configuró un pseudo entorno (Lippmann, 1922) "postpandémico", de escasez de información sobre la enfermedad, justo en el pico de su evolución objetiva. De este modo, sobrerrepresentación, polarización e invisibilización constituyeron las tres fases del ciclo de mediatización de la pandemia en los principales medios digitales de la Argentina. 
Los resultados de este trabajo pretenden dar cuenta de un proceso que es dinámico y permanece abierto. La novedad y gravedad de la situación que atraviesa el mundo invita a repensar la productividad de ciertas hipótesis y a formular otras que permitan acceder científicamente a un escenario social y comunicacional inédito. Los hallazgos empíricos dan cuenta de rupturas y continuidades. Entre las primeras, resulta necesario revisitar los postulados sobre la relación entre fuentes oficiales y medios, sobre todo en contextos de catástrofe y en el marco de un ecosistema de medios convergentes que favorece la desinformación. Entre las segundas, la constatación de la existencia, aún en pandemia, de coberturas polarizadas que simplifican el debate público invitan a pensar los procesos de sobreinformación y desinformación más allá de los medios conectivos, aunque en relación con estos. Los aportes quedan abiertos, entonces, para nuevos estudios comparativos y multidimensionales a nivel nacional e internacional.

\section{Bibliografía}

Bagdikian, B.H. (1985). The U. S. Media Supermarket or Assembly Line? Journal of Communication, 3(35), 97-109. https://doi.org/10.1111/j.1460-2466. 1985.tb02451.x

Beck, U. (1998). La sociedad del riesgo. Hacia una nueva modernidad (1. ${ }^{\text {a ed.). }}$ Paidós Ibérica.

Bennett, W.L., \& Livingston, S. (2018). The disinformation order: Disruptive communication and the decline of democratic institutions. European Journal of Communication, 33(2), 122-139. https://doi.org/10.1177/02673231 18760317

Bennett, W.L. (1990). Toward a Theory of Press-State Relations in the United States. Journal of Communication, 40(2), 103-127. https://doi.org/10.1111/j. 1460-2466.1990.tb02265.x

Bennett, W.L., \& Lawrence, R.G., \& Livingston, S. (Eds.) (2007). When the Press Fails. Political Power and The News Media from Irak to Katrina. 1. ${ }^{a}$ ed. The University of Chicago Press.

Brosius, H.B., \& Eps, P. (1995). Prototyping through Key Events: News Selection in the Case of Violence Against Aliens and Asylum Seekers in Germany. European Journal of Communication, 10(3), 391-412. https://doi. org/10.1177/0267323195010003005 
Budd, R.W. (1964). U.S. News in the Press Down Under. The Public Opinion Quarterly, 28(1), 39-56. https://bit.ly/3oH1tKS

Cappella, J.N., \& Jamieson, K.H. (1996). News Frames, Political Cynicism, and Media Cynicism. Annals AAPSS, 546, 71-84. https://bit.ly/3roR5cL

Casero-Ripollés,A.(2020). Impact of COVID-19 on the media system.Communicative and democratic consequences of news consumption during the outbreak. Profesional de la Informacion, 29(2), 1-11. https://doi.org/10.3145/epi. 2020.mar.23

Charron, J. (1995). Los medios y las fuentes. Los límites del modelo de agenda setting. En M. J. Gilles Gauthier (Ed.), Comunicación y Política (1º, pp. 72-93). Gedisa.

Duc-Huynh, T.L. (2020). The COVID-19 risk perception: A survey on socioeconomics and media attention. Economics Bulletin, 40(1), 758-764.

Farré-Coma, J. (2015). Comunicación de riesgo y espirales del miedo. Comunicación y Sociedad, O(3), 95-119. https://doi.org/10.32870/cys.v0i3.4209

Ferree, M.M., Gamson, W.A., Gerhards, J., \& Rucht, D. (2002). Shaping Abortion Discourse. Democracy and the Public Sphere in Germany and the United States. Cambridge.

Gans, H. (1979). Deciding What's News (1. a ed.). Northwestern University Press.

Hallin, D. C., Briggs, C. L., Mantini-Briggs, C., Spinelli, H., \& Sy, A. (2020). Mediatización de las epidemias: la cobertura sobre la pandemia de la gripe A (H1N1) de 2009 en Argentina, Estados Unidos y Venezuela. Comunicación y Sociedad, e7207, 1-24. https://doi.org/10.32870/cys.v2020.7207

Jablonski, P., \& Daniele, J. (1998). Variation on Issue Obstrusiveness and its Impact on Agnda Building: The Case of Base Realignment and Closing. World Communication, 27(3), 31-46.

Kessler, G., \& Focás, B. (2014). ¿Responsables del temor? Medios y sentimiento de inseguridad en América Latina. Nueva Sociedad, 249, 137-148. https:// bit.ly/3pFKN83

Koziner, N. (2018). Standing de las fuentes periodísticas en la política de medios argentina (2009 y 2016). Cuadernos Info, 42, 141-158. http://dx.doi. org/10.7764/cdi.42.1291

Krippendorff, K. (1990). Metodología de análisis de contenido. Teoría y práctica (1. a ed.). Paidós.

Lee, G. (2004). Reconciling «Cognitive Priming» vs «Obtrusive Contingency» Hypotheses: An Analytical Model of Media Agenda-Setting Effects. Inter- 
national Communication Gazette, 66(2), 151-166. https://doi.org/10.11 77/0016549204041475

Lippmann, W. (1922). Public Opinion (1. a ed.). Harcour, Brace and Company Inc. Luhmann, N. (1973). Ilustración sociológica y otros ensayos (1. a ed.). Editorial Sur. Martini, S. (2017). Últimas noticias. Construyendo la actualidad en el siglo XXI. En S. Martini \& M. Pereyra (Eds.), La noticia hoy. Tensiones entre la política, el mercado y la tecnología (1. a ed., pp. 1-28). IMAGO MUNDI.

Masip, P., Aran-Ramspott, S., Ruiz-Caballero, C., Suau, J., Almenar, E., \& PuertasGraell, D. (2020). Consumo informativo y cobertura mediática durante el confinamiento por la COVID-19: sobreinformación, sesgo ideológico y sensacionalismo. El profesional de la información, 29(3), 1-12. https:// bit.ly/3j8odm7

McCombs, M. (2006). Estableciendo la agenda. El impacto de los medios en la opinión pública y el conocimiento (1. ${ }^{\mathrm{a}}$ ed.). Paidós Ibérica.

McCombs, M., \& Valenzuela, S. (2014). Agenda-Setting Theory: The Frontier Research Questions (Número May 2018). https://doi.org/10.1093/oxfordhb/9780199793471.013.48

Mitchelstein, E., \& Boczkowski, P.J. (2017). Juventud, estatus y conexiones. Explicación del consumo incidental de noticias en redes sociales. Revista Mexicana de Opinión Pública, 24, 131. https://doi.org/10.22201/ fcpys.24484911e.2018.24.61647.

Muñiz, C. (2020). Media System Dependency and Change in Risk Perception During the COVID-19 Pandemic 11. Tripodos, 1(47), 11-26. https://bit.ly/3p GRGG1

Muñiz, C., \& Corduneanu, V. I. (2020). Percepción de riesgo y consumo mediático durante el inicio de la pandemia de COVID-19 en México. Más Poder Local, 41(abril), 19-22. https://bit.ly/3askStQ

Neuendorf, K. (2002). The content analysis guidebook (1. ${ }^{\text {a }}$ ed.). Sage.

Palpan-Guerra, A., \& Munayco, C. V. (2015). How did the media report on the AH1N1 influenza in Peru? Revista Peruana de Medicina Experimental y Salud Publica, 32(2), 294-298. https://doi.org/10.17843/rpmesp.2015. 322.1623

Roberts, C. (2005). Gatekeeping theory: An evolution. Channels, 17. https://bit. ly/2YD0nFi

Rodrigo-Alsina, M. (1989). La producción de la noticia. En La construcción de la noticia (1. ${ }^{\mathrm{a}}$ ed., pp. 140-200). Paidós. 
Salaverría, R., Buslón, N., López-Pan, F., León, B., Erviti, M., López-Goñi, I., \& Erviti, M.C. (2020). Desinformación en tiempos de pandemia: tipología de los bulos sobre la COVID-19 Disinformation in times of pandemic: typology of hoaxes on COVID-19. El profesional de la información,29(3), 1-15. https://bit.ly/2MHSvzO

Schlesinger, P. (1992). Repensando la sociología del periodismo. Esrategias de las fuentes y límites del centralismo en los medios. Estudios sobre las culturas contemporáneas, IV(13-14), 279-307. https://bit.ly/3pG6JQf

Thompson, K. (2014). Pánicos morales (1. ${ }^{\mathrm{a}}$ ed.). Universidad Nacional de Quilmes Editorial.

Zunino, E.A. (2019). ¿Quién define la agenda ? Las fuentes de información en la prensa digital argentina. Comunicacion y Sociedad, e7394, 1-23. https:// doi.org/https://doi. org/10.32870/cys.v2019i0.7394

Zunino, E., \& Grilli Fox, A. (2020). Medios digitales en la Argentina: posibilidades y límites en tensión. Estudios sobre el Mensaje Periodístico, 26(1), 401413. https://doi.org/10.5209/esmp.67320

Fecha de envío: 2020/10/30; Fecha de aceptación: 2021/01/28;

Fecha de publicación: 2021/03/01 